\title{
Characterization and imaging of surgical specimens of invasive breast cancer and normal breast tissues with the application of Raman spectral mapping: A feasibility study and comparison with randomized single-point detection method
}

\author{
HAIPENG ZHANG ${ }^{1 *}$, XIAOZHEN WANG ${ }^{2 *}$, RONGBO DING ${ }^{2}$, LISHENGNAN SHEN $^{2}$, PIN GAO $^{2}$, \\ HUI XU ${ }^{3}$, CAIFENG XIU ${ }^{1}$, HUANXIA ZHANG ${ }^{1}$, DONG SONG ${ }^{2}$ and BING HAN ${ }^{2}$ \\ Departments of ${ }^{1}$ Gynaecology, ${ }^{2}$ Breast Surgery and ${ }^{3}$ Ophthalmology, \\ The First Hospital of Jilin University, Changchun, Jilin 130021, P.R. China
}

Received April 24, 2019; Accepted January 24, 2020

DOI: $10.3892 / \mathrm{ol} .2020 .11804$

\begin{abstract}
A mapping technique was used in the present study to explore the biological and imaging characteristics of invasive breast cancer and normal breast tissues in Raman examination data and construct a diagnostic model for breast cancer. Raman examination data reflect the biochemical or molecular characteristics of the target tissues. A total of 45 specimens from patients with breast cancer who underwent surgery and 25 adjacent normal breast tissue specimens were included in the present study. Using the specimens, a total of 53 sets of mapping data and 2,597 pieces of Raman spectral data were obtained. The collected spectra were corrected and fitted, the Raman spectra were analyzed by robust statistical methods, and a diagnostic model was constructed using the k-Nearest Neighbor (KNN) method. The KNN classification method was applied to analyze the characteristics of the mapping test application. The percentage of outliers in the mapping data for malignant and normal breast tissues was 12.7 and $6.6 \%$, respectively. The percentage of outlier data in the conventional single-point detection data for malignant and normal breast tissues was 24.5 and $26.0 \%$, respectively. Analysis using a t-test identified a significant difference in the number of outliers between mapping and single-point detection for malignant $(\mathrm{t}=-6.169 ; \mathrm{P}<0.001)$ and normal breast tissues $(\mathrm{t}=-8.873 ; \mathrm{P}<0.001)$. Based on the mapping data, the accuracy, sensitivity and specificity for breast cancer detection
\end{abstract}

Correspondence to: Dr Bing Han, Department of Breast Surgery, The First Hospital of Jilin University, 71 Xinmin Street, Changchun, Jilin 130021, P.R. China

E-mail: han_bing@jlu.edu.cn

${ }^{*}$ Contributed equally

Key words: bioimaging, mapping, intraoperative, model building, breast cancer, Raman spectroscopy by the diagnostic model constructed using the KNN method was $99.56,96.6$ and $98.48 \%$, respectively. The positive and negative predictive value of this model was 99.56 and $89.04 \%$, respectively. The data obtained by mapping technology demonstrated improved stability and contained less outliers compared with single-point detection. The diagnostic model constructed using the mapping data demonstrated excellent diagnostic performance and good correspondence with pathological results. The findings of the present study demonstrated the feasibility of the application of the diagnostic model for intraoperative real-time imaging for patients with breast cancer. This study provided the foundation of Raman spectroscopy-based diagnostic imaging at the molecular level.

\section{Introduction}

Breast-conserving surgery (BCS) for patients with breast cancer is gaining popularity due to the comparable survival outcomes of BCS and modified radical mastectomy (MRM) demonstrated by several studies (1). Currently, BCS accounts for $\sim 60 \%$ of all breast cancer surgeries in the USA compared with $\sim 20 \%$ in China (2,3). The most important aspect of $\mathrm{BCS}$ is complete resection of the lesion area to ensure that the surgical margin is negative as a positive surgical margin means a higher local recurrence rate. At present, there is a lack of a rapid and accurate diagnostic methods for breast cancer and pathological diagnosis is the most common method used in clinical settings (4). However, histopathological examination of paraffin sections is a time- and resource-intensive procedure. In addition, it does not meet the requirements of rapid diagnosis and may result in a higher rate of secondary surgery (5). Intraoperative frozen section pathology is relatively fast; however, the diagnostic accuracy is limited due to the small amount of obtained tissues (6). Thus, the development of a rapid, convenient and accurate diagnostic method is required to improve outcomes of BCS and to minimize the chances of secondary surgery.

Raman spectra are generated by inelastic scattering of photons on the surface of molecules. Each molecule has a set 
of energy levels, and the scattered spectrum is dependent on the energy transfer between the molecule and the photons. By analyzing the relative strength and position of each characteristic peak in the Raman scattering spectrum, the biochemical composition information of the sample can be obtained (7). Raman spectroscopy is often used for the detection and imaging of crystals $(8,9)$. Raman spectroscopy diagnosis at the molecular level offers the advantage of possible detection of lesions prior to the development of overt morphological changes; therefore, it may facilitate identification of the tumor edge and help reduce the local recurrence rate (10). Raman spectra can provide molecular vibration and rotation information $(11,12)$. Raman spectroscopy is a simple, fast and sensitive method that can directly analyze tissues without any preprocessing and offers the potential for rapid diagnosis of pathological features of tissues during surgery (13). Raman spectroscopy is promising for clinical application and has become a research hotspot for rapid diagnosis of breast tumors (14-17). Raman spectroscopy identifies molecular vibrations and so allows breast cancer tissue to be distinguished from surrounding normal tissue using mathematical processing and computer modeling (18). The majority of recent studies have generated data based on random single-point detection or targeted random-point detection $(12,19)$. Some researchers have used surface enhanced Raman spectroscopy and other surface enhancement methods to enhance the signal to obtain improved results (20).

For unprocessed fresh breast cancer tissues, the heterogeneity of the biochemical components in the tissues and the blindness of the detection location lead to the great difference in the results of the Raman data in the detected tissues; it is difficult to analyze such results and construct diagnostic models $(14,21)$, even with use of mathematical methods such as linear regression, partial least squares and the artificial neural network. During the test, a laser beam is emitted from the microscope of the Raman instrument. Since the laser detection point is very small, the beam may focus on the nucleus, the cytoplasm, an organelle or the interstitium during the detection process, thus not achieving the ideal result (21). The peak assignment and difference of Raman spectra between cancer and normal tissues have been explored and described by numerous studies $(14,15,22,23)$. Raman data of single-point detection may be used to identify benign and malignant breast cancer; however, it can only provide chemical information about the tissue. Since the obtained tissue often contains a number of different components with similar spectral characteristics, it is difficult to provide reliable information about the tissue structure (15). The Raman spectroscopy mapping method can provide information pertaining to the chemical composition as well as information about specific areas by helping identify specific areas of the tissue. The analysis results obtained using mapping display the morphological structure inside the tissue and the spatial distribution of the relative content of biochemical components, such as DNA and actin $(24,25)$. Yu et al (25) successfully identified normal cells and cancer cells by using Raman mapping spectra, which indicated the feasibility of using the mapping technique for the detection of breast cancer tissues.

Breast tissue is inherently heterogeneous. The fingerprint characteristics of Raman spectra also enable the analysis of Raman spectral data of breast cancer tissues to provide in-depth information on the malignant transformation process of breast tissues. Several research groups investigated the effectiveness of Raman imaging in clinical diagnosis $(13,24)$. However, Raman spectroscopy has not been used to image breast tissue. Our earlier studies showed that the single point detection technique of Raman spectrum could distinguish normal breast tissue, breast cancer tissue and benign breast tissue (15). However, due to the heterogeneity of tissues and detection methods, the obtained data varied greatly and showed poor regularity. In order to improve the stability of Raman data obtained in the present study, Raman spectrum mapping detection was used to minimize the influence of organizational structure and human factors, for example slight movement of the microscope lens after fixation, on the results. This technique was used to construct a breast cancer diagnostic model to conduct imaging analysis and create pseudocolor Raman maps. A map similar to the pathological hematoxylin and eosin (H\&E) images were obtained, demonstrating the reliability of Raman spectroscopy method for tissue imaging. The results of the present study may inform future studies investigating real-time imaging of incisor edge lesions.

\section{Materials and methods}

Patient information. A total of 45 breast cancer tissue specimens from patients who underwent MRM or simple mastectomy at the First Hospital of Jilin University (Changchun, China) between July 2015 and January 2016 were used in the present study. Of these, 22 tissue specimens were subjected to mapping and 23 were subjected to random single-point Raman spectroscopy. A total of 25 samples of adjacent normal breast tissues were also collected. Mapping and random-single point detection was performed in 15 and 10 normal breast specimens, respectively. All patients were female with a median age of 52 years (age range, 32-63). The only inclusion criterion for the present study was invasive breast cancer. The exclusion criteria were patients who refused to participate in the trial. Following further examination by preoperative biopsy and postoperative pathology, the breast tissues were confirmed as invasive ductal carcinoma; however, breast cancer stage was not assessed). All patients agreed to participate in the study and provided written informed consent. The study protocol was approved by the Ethics Committee of the First Hospital of Jilin University (approval no. 2013-168).

Specimen collection. Breast cancer tissue and adjacent normal breast tissue (as far away as possible from the lesion, $\geq 5 \mathrm{~cm}$ ) of the same patients were collected, and the adipose tissues were removed. The specimens were immediately frozen at -25 to $-20^{\circ} \mathrm{C}$. Subsequently, two contiguous sections were sliced using a freezing microtome (cat. no. CM3050S; Leica Microsystems $\mathrm{GmbH}$ ). Following this, the specimens were stained with $\mathrm{H} \& \mathrm{E}$ for further examination. $\mathrm{H} \& \mathrm{E}$ staining was performed as follows. First, the specimens were immersed in $10 \%$ neutral buffered formalin for $12 \mathrm{~h}$ at room temperature and then dehydrated in 75, 80, 95 (I), 95 (II), 100 (I) and $100 \%$ (II) ethanol for $1 \mathrm{~h}$, respectively. Next, specimens were washed in in xylene for $1 \mathrm{~h}$ twice, paraffin embedded for $4 \mathrm{~h}$, 


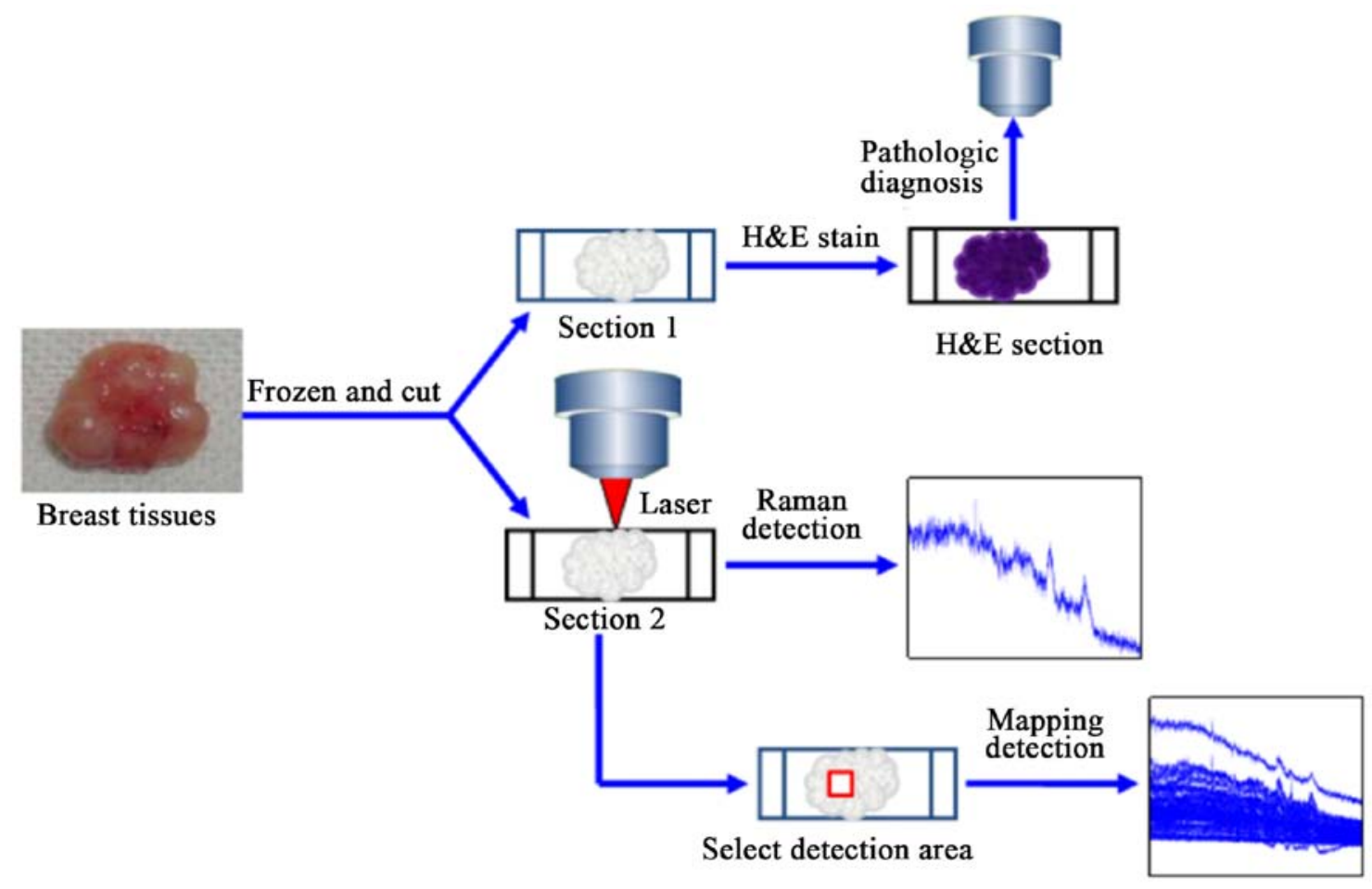

Figure 1. Schematic representation of the detection procedure of breast tissues by Raman spectroscopy and histopathology. H\&E, hematoxylin and eosin.

and then cut into 3-5 $\mu \mathrm{m}$-thick sections. H\&E staining was performed at $22^{\circ} \mathrm{C}$ with $\mathrm{H}$ staining for $5 \mathrm{~min}$, then sections were washed with running water for $1 \mathrm{~min}$, dipped in $0.1 \%$ $\mathrm{HCl}$ for $10 \mathrm{sec}$ and $\mathrm{E}$ counterstained for $1 \mathrm{~min}$. Stained sections were then dehydrated in 75, 80, 95 (I), 95 (II), 100 (I) and $100 \%$ (II) ethanol for $1 \mathrm{~min}$, respectively. Finally, specimens were dipped in xylene for 1 min three times. Dried sections were sealed with neutral gum. In the process of making pathological sections, one of the two adjacent sections was stained with H\&E for routine histopathological analysis by two experienced breast pathologists, and the other section was transported in liquid nitrogen for Raman spectroscopy without any further processing. To ensure that the detection area was malignant, the H\&E-stained section was used as a guide to determine the detection area of the frozen section.

Raman spectroscopy. Raman spectroscopy was performed using a confocal Raman System (HORIBA) (http://www.horiba.com/en_en/) at the State Key Laboratory of Supramolecular Structures and Materials of Jilin University (Changchun, China). It is a combination of a Raman spectrometer and a standard optical microscope, with an optical microscope at the bottom for image acquisition and a Raman spectrometer at the top. The optical microscope is used to capture images of the area being examined, and the laser beam excited by the instrument is focused through the optical microscope as a tiny spot of light with a diameter of $1.5 \mu \mathrm{m}$. The Raman signal in the area where the spot is located passes through the microscope back to the Raman spectrometer to obtain the Raman spectral information of the tissue. For the single point test, a $633 \mathrm{~nm}$ helium-neon laser was used in Duoscan mode, and the selected tissue area was scanned point by point. The Raman signal generated during the test was detected by a Synapse Thermoelectric cooled charge-coupled device camera (Horiba Jobin Yvon SAS) with a spatial resolution of $3 \lambda$. The power of the laser reaching the tissue surface was $20 \mathrm{~mW}$. No photo damage was observed in the samples after the mapping data acquisition. Rayleigh scattered light was filtered using a 4-notch filter (Horiba Jobin Yvon). The scanning range was $400-3,000 \mathrm{~cm}^{-1}$, the integration time was $20 \mathrm{sec}$ and the number of integration times was 1 . The test tissue was kept moist with saline to effectively reduce the spectral background and photodegradation. Prior to the Raman spectroscopy test, images of the H\&E-stained sections of the breast tissue were captured using a light microscope (Olympus Corporation) at $\mathrm{x} 10$ and x50 magnification . The optical images were obtained at the same position as the corresponding continuous frozen section. The wave number calibration setting referred to the vibration frequency of the silicon wafer at $520.7 \mathrm{~cm}^{-1}$, and these parameters remained unchanged during all measurement processes (Fig. 1).

Data collection. From the H\&E-stained sections, representative regions of malignant and normal cells were selected for Raman spectroscopy on frozen contiguous sections. During the collection of single-point Raman spectra of tissues, 20-30 spectra were obtained in each sample from different locations to ensure representative sampling and collection of varying signals. During collection of the mapping spectrum, each section was scanned for 1-3 regions to obtain a Raman map of this region by point-to-point detection; a total of $7 \times 7$ points were scanned.

Data processing. A total of 53 sets of mapping data and 2,597 single-point Raman spectra were obtained for detection (each 
Table I. Outlier data of the mapping test.

\begin{tabular}{|c|c|c|c|c|}
\hline \multirow{2}{*}{$\begin{array}{l}\text { Classification } \\
\text { group }\end{array}$} & \multicolumn{2}{|c|}{$\begin{array}{l}\text { Mapping of breast } \\
\text { tissue (Outlier data) }\end{array}$} & \multicolumn{2}{|c|}{$\begin{array}{l}\text { Single point detection of } \\
\text { breast tissue (Outlier data) }\end{array}$} \\
\hline & Malignant & Normal & Malignant & Normal \\
\hline 1 & 7 & 2 & 9 & 13 \\
\hline 2 & 2 & 2 & 10 & 15 \\
\hline 3 & 5 & 1 & 15 & 12 \\
\hline 4 & 6 & 4 & 12 & 8 \\
\hline 5 & 8 & 9 & 5 & 17 \\
\hline 6 & 6 & 6 & 14 & 13 \\
\hline 7 & 5 & 2 & 15 & 15 \\
\hline 8 & 7 & 2 & 11 & 12 \\
\hline 9 & 9 & 2 & 14 & 10 \\
\hline 10 & 6 & 2 & 12 & 10 \\
\hline 11 & 6 & 1 & 7 & 15 \\
\hline 12 & 5 & 2 & 16 & - \\
\hline 13 & 8 & 7 & 13 & - \\
\hline 14 & 4 & - & 15 & - \\
\hline 15 & 6 & - & - & - \\
\hline 16 & 6 & - & - & - \\
\hline 17 & 7 & - & - & - \\
\hline 18 & 6 & - & - & - \\
\hline 19 & 7 & - & - & - \\
\hline 20 & 7 & - & - & - \\
\hline 21 & 10 & - & - & - \\
\hline 22 & 6 & - & - & - \\
\hline 23 & 5 & - & - & - \\
\hline 24 & 5 & - & - & - \\
\hline
\end{tabular}

set of mapping data comprised 49 spectra). Among these, 34 sets were form malignant tissues with a total of 1,666 spectra and 19 sets were from normal breast tissue with a total of 931 spectra. A total of 1,280 Raman spectra were obtained from single-point detection including 720 from malignant tissues and 560 from benign tissues. The spectra were subjected to baseline correction by fitting and subtracting a trinomial polynomial using NGSLabSpec version 5.58.25 software (Horiba, Ltd.). The spectral data was then smoothed using a 15-point adjacent averaging algorithm. Average spectra of Raman data were calculated using Matlab version 7.9.0 software (The MathWorks, Inc.).

$k$-Nearest Neighbor (KNN) method. KNN algorithm is run by self-programming program in MATLAB R2009b software (https://ww2.mathworks.cn/products/matlab.html), used to classify Raman data (26). By finding the $\mathrm{k}$ closest neighbors of the Raman characteristic peaks of the breast tissue and assigning the average properties of these neighbors to the sample, the characteristics of breast tissue in different lesions can be analyzed.

Mapping imaging method. A total of 24 sets (70.6\%) of malignant data were used to construct the diagnostic model and mapping imaging and 10 sets $(29.4 \%)$ were used to test the model. The diagnostic model was also constructed and tested in 13 sets $(68.4 \%)$ and 6 sets $(31.6 \%)$ of normal breast tissues. Single-point test results were used in the malignant group and the normal breast tissue group using 504 and 392 spectra (70\%), respectively, as training sets for construction of the models; 216 and 168 spectra (30\%), respectively, were used as test sets for testing the models and analysis. t-tests (SPSS.20.0) were applied to analyze 2 sets of data. Raman spectroscopy images were analyzed by the KNN classification method and processed by diagnostic models to explore the advantages and feasibility of practical application.

\section{Results}

Mapping is more stable compared with single-point detection due to fewer outliers. Mapping data is more conducive to building a model with high discrimination efficiency. Table I presents the number of outliers in the measured data of each group; greater the outlier number indicates lower stability. The mapping data for malignant breast tissues consisted of 24 sets. The malignant outlier data comprised 149 pieces [mean There were 140 pieces of outlier data [average, 12.7 (8-17) pieces/group; Table I]. A significant difference was observed in the number of outliers between mapping and single-point detection data in the malignant group $(\mathrm{t}=-6.169 ; \mathrm{P}<0.001)$. A 
Table II. Diagnostic results of the k-Nearest Neighbor model.

\begin{tabular}{|c|c|c|c|c|c|}
\hline Variable & $\mathrm{OA}$ & SE & SP & PPV & NPV \\
\hline Mapping of malignant breast tissue, $\%$ & 99.56 & 96.60 & 98.48 & 99.56 & 89.04 \\
\hline Mapping of normal breast tissue, $\%$ & 89.04 & & & & \\
\hline Single-point detection of malignant breast tissue, $\%$ & 90.70 & 84.78 & 50.00 & 90.70 & 63.64 \\
\hline Single-point detection of normal breast tissue, $\%$ & 63.64 & & & & \\
\hline
\end{tabular}

OA, overall accuracy; SE, sensitivity; SP, specificity; PPV, positive predictive value; NPV, negative predictive value .
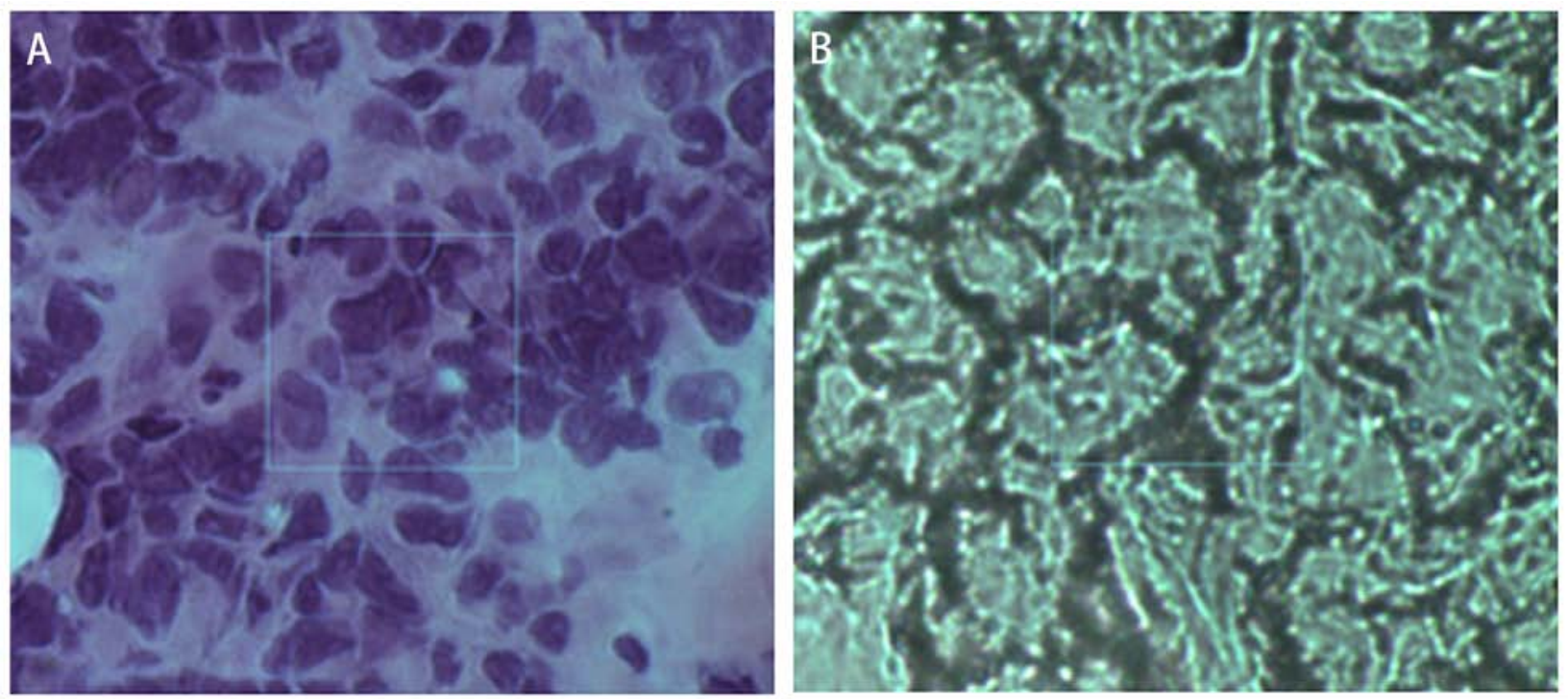

Figure 2. H\&E-stained and unstained contiguous pathology section (magnification, x50). The square frame represents the testing area. In mammary gland tissue, the cancer cells are disordered and have large atypia. In H\&E sections, nucleic acids in chromatin and cytoplasm are purplish blue, and components in cytoplasm and extracellular matrix are red. (A) H\&E-stained section; (B) frozen section without staining. H\&E, hematoxylin and eosin.

similar result was obtained for normal breast tissues $(\mathrm{t}=-8.873$; $\mathrm{P}<0.001)$.

Mapping is more robust compared with single-point detection according to the diagnostic model. The evaluation indicators were overall accuracy (OA), sensitivity (SE), specificity (SP), positive predictive value (PPV) and negative predictive value (NPV). The results of the five evaluation indicators obtained from the test set are presented in Table II. The correct OA rates for breast cancer and normal breast tissue prediction were 99.56 and $89.04 \%$, respectively. The overall SE, SP, PPV and NPV of the mapping data diagnostic model were 96.60, $98.48,99.56$ and $89.04 \%$, respectively. With single-point data, the correct OA rates for breast cancer and normal breast tissue prediction were 90.70 and $63.64 \%$, respectively. The overall SE, SP, PPV and NPV of the diagnostic model were 84.78, $50.00,90.70$ and $63.64 \%$, respectively (Table II).

KNN classification imaging map. Blue and green represented malignant and non-malignant tissues in the map, respectively. The map was restored according to the coordinates of each detection point (Figs. 2 and 3). Fig. 2 shows the comparison between H\&E stained pathological sections and unstained continuous pathological sections. In mammary gland tissue, the cancer cells are disordered and have large atypia. By observing the H\&E section, the cell structure can be better observed. Fig. 2A is the section stained by H\&E, and Fig. 2B is the frozen section. The location shown in the box in the figure is the cancerous region. The large blue area corresponded to the nucleus of the pathological section, whereas the green area corresponded to the tumor stroma (Fig. 3). The images of this method were consistent with the distribution of tumor and interstitial cells on pathological sections.

\section{Discussion}

The percentage of BCS is increasing yearly (27). The aim of $\mathrm{BCS}$ is to achieve negative margins to avoid re-excision and achieve good esthetic results (28). The results reported at the 2015 San Antonio breast cancer conference revealed that $\sim 17 \%$ of patients underwent secondary surgery following BCS (5). Clinicians and researchers have been exploring ways to reduce the rate of secondary surgery through preoperative examination (29). There are many methods to evaluate the surgical margin of breast-conserving surgery, including clinical observation and palpation, in vivo or specimen imaging examination and pathological evaluation. A recent meta-analysis showed that the sensitivity 

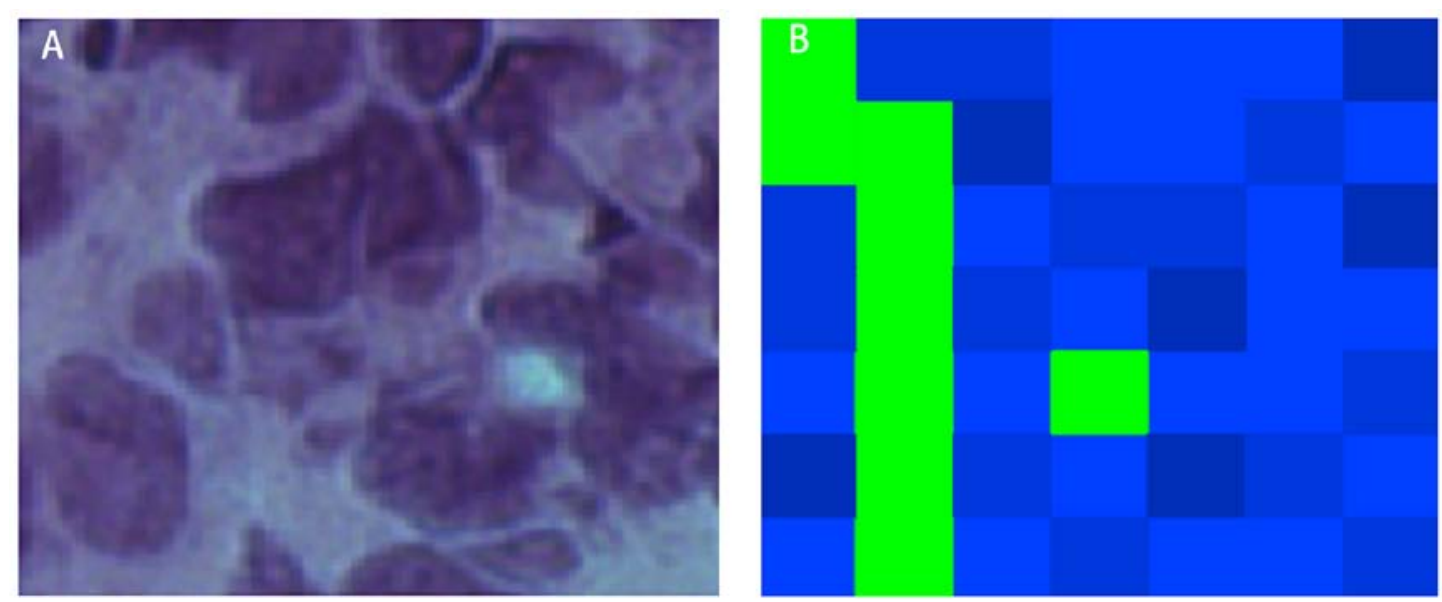

Figure 3. Comparison of the testing area on (A) the pathology image (magnification of figure $2 \mathrm{~A}, \mathrm{x} 50$ ) and (B) the mapping image. In (B), the large blue area corresponded to the nucleus of the pathological section, whereas the green area corresponded to the tumor stroma. In addition, the blue represents the malignant areas identified using the Raman spectroscopy diagnostic model that we build by KNN method and green represents the areas that are non-malignant.

and specificity of intraoperative ultrasound examination of the surgical margin of the tumor were 59 and $81 \%$, respectively, and the sensitivity and specificity of specimen X-ray examination were 53 and $84 \%$, respectively, which did not significantly reduce the incidence of secondary surgery (30). Thus, it is imperative to introduce a new technique for the detection of the surgical margin in BCS. Surgeons require a quick, comprehensive test to determine whether there are any residual cancer cells at the surgical margin. Indocyanine green and microwave technologies are ineffective at reducing the rate of secondary surgery following BCS $(31,32)$. At present, the most popular methods for the determination of the surgical margin or tissue properties include Raman spectroscopy and real-time imaging of tumor cells with fluorescent nanoparticles $(15,33)$.

In the present study, a mapping technique of Raman spectroscopy was used to minimize the influence of tissue heterogeneity and inter-observer variability on the results. The detection method of the mapping technology involves fixed interval and point-by-point scanning per unit area; therefore, the results are more objective and stable. However, mapping is relatively time-consuming. H\&E-stained malignant and normal breast tissue sections were used to identify areas with the most abundant cells; areas with a size of 10x10 $\mu \mathrm{m}$ were selected, and the corresponding positions on the frozen sections were identified for detection. In the mapping data set, the average number of outliers in malignant tissues was 6.2 pieces/group, which accounted for $12.7 \%$ of the total training set data; in the conventional single-point detection, the average number of outliers in the malignant group were 12 pieces/group, which accounted for $24.5 \%$ of all data. A significant difference between outlier numbers was observed between the two techniques. The data obtained in the present study demonstrated that the mapping method was more stable compared with single-point detection and contains fewer outliers.

Mapping data are more conducive for construction of diagnostic models (25). Robust statistics is a statistical method that minimizes the effect of extreme results on the mean and standard deviation estimates (21). An in-depth discussion on the application of robust statistics was provided in our previous study (12). However, robust statistics is not relevant if the data is too discrete (21). In the present study, the number of outliers was small and the construction model could obtain effective spectral results, which was conducive to model construction.

In the present study, the KNN method was used to construct a diagnostic model based on robust statistics. The diagnostic accuracy for malignant tumors was $99.56 \%$, and the NPV was $89.04 \%$, which was significantly higher compared with single-point test results. The diagnostic model was also superior to the results reported in previous studies $(16,17)$ with respect to accuracy and specificity; this further verified the advantages of the mapping method for construction of breast cancer diagnostic models. The present study differed from previous studies $(17,25)$ in that the training data for the model construction was not used to test the model; instead, new data were used, which reduced false high accuracy due to the same training and testing data. The present study identified new possibilities for sensitive detection of the tumor margins at the level of mapping molecules. Although limited reports have been published on the use of this method for detection of breast cancer tissue, it has recently become a new research hotspot $(15,17)$.

In the present study, a diagnostic model was used to evaluate the images. The imaging method for classification by the diagnostic model must be based on a good classification model. Behl et al (13) performed Raman spectroscopy imaging of the oral mucosa. The data was de-baselined and then analyzed by the K-means cluster analysis method; tumor, stroma and inflammatory cells were assigned different colors (red, green and blue), and these regions are distinctly depicted on Raman maps of tumor sections (13). Daniel et al (34), used a combination of principal component analysis and $\mathrm{K}$ classification to image the oral mucosa and classify the different principal components. In the present study, a breast cancer diagnosis model was constructed by KNN method, and the Raman map obtained by imaging the detected area could clearly depict the tumor margins. This correspondence demonstrated the application value of this method for real-time imaging of tumor margins. In the future, real-time 
margin imaging by software determined properties may be used to guide surgical resection.

In conclusion, confocal micro-Raman spectroscopy was used to detect the features of breast cancer and normal breast tissue by point-by-point scanning, and the results of the present study demonstrated that it was possible to obtain more stable spectral data compared with the data obtained using previous methods. Based on the spectral data, the KNN method was used to construct the diagnostic model software. The diagnostic accuracy was significantly higher compared with that of the model built using the single-point detection method, which demonstrated the advantage of the mapping method for data acquisition. The image obtained using the imaging method based on the diagnostic model classification closely corresponded to the pathological sections, which revealed the feasibility of the application of mapping for intraoperative imaging. The present study provided a foundation for the diagnostic use of the Raman spectrum at the molecular level.

\section{Acknowledgements}

Not applicable.

\section{Funding}

The current study was supported by a grant from the National Nature Foundation of China (grant no. 81773171).

\section{Availability of data and materials}

The datasets used and/or analyzed during the present study are available from the corresponding author on reasonable request.

\section{Authors' contributions}

HPZ, XZW, DS and BH contributed to the conception of this study and performed the preliminary documentation. RBD, LS, PG, HX, CFX and HXZ enrolled patients in the study and collected clinical data. All authors participated in the statistical analysis and contributed to the interpretation of the results as well as drafting the manuscript. All authors have read and approved the manuscript.

\section{Ethics approval and consent to participate}

This research followed the International and National regulations in accordance with the Declaration of Helsinki. The study was approved by the Ethics Committee of the First Hospital of Jilin University (Changchun, China). All patients provided written informed consent before being included in this study.

\section{Patient consent for publication}

Not applicable.

\section{Competing interests}

The authors declare that they have no competing interests.

\section{References}

1. Fisher B, Anderson S, Bryant J, Margolese RG, Deutsch M, Fisher ER, Jeong JH and Wolmark N: Twenty-year follow-up of a randomized trial comparing total mastectomy, lumpectomy, and lumpectomy plus irradiation for the treatment of invasive breast cancer. N Engl J Med 347: 1233-1241, 2002.

2. Yiannakopoulou EC and Mathelin C: Oncoplastic breast conserving surgery and oncological outcome: Systematic review. Eur J Surg Oncol 42: 625-630, 2016

3. Rubio IT, Ahmed M, Kovacs T and Marco V: Margins in breast conserving surgery: A practice-changing process. Eur J Surg Oncol 42: 631-640, 2016.

4. Wen HY and Brogi E: Breast cancer pathology. Oncoplastic Reconstructive Br Surg 7: 87-127, 2019.

5. Bodilsen A, Bjerre K, Offersen BV, Vahl P, Amby N, Dixon JM, Ejlertsen B, Overgaard $\mathrm{J}$ and Christiansen P: Importance of margin width in breast-conserving treatment of early breast cancer. J Surg Oncol 113: 609-615, 2016.

6. Eskelinen M, Collan Y,Puittinen J and Valkamo E: Frozen section diagnosis of breast cancer. Acta Oncologica 28: 183-186, 1989.

7. Downes A and Elfick A: Raman spectroscopy and related techniques in biomedicine. Sensors (Basel) 10: 1871-1889, 2010.

8. Furuyama N, Hasegawa S, Hamaura T, Yada S, Nakagami H, Yonemochi E and Terada K: Evaluation of solid dispersions on a molecular level by the Raman mapping technique. Int J Pharm 361: 12-18, 2008.

9. Ueda H, Ida Y, Kadota K and Tozuka Y: Raman mapping for kinetic analysis of crystallization of amorphous drug based on distributional images. Int J Pharm 462: 115-122, 2014.

10. Zhang R, Zhang Y, Dong ZC, Jiang S, Zhang C, Chen LG, Zhang L, Liao Y, Aizpurua J, Luo Y, et al: Chemical mapping of a single molecule by Plasmon-enhanced Raman scattering. Nature 498: 82-86, 2013.

11. Hanlon EB, Manoharan R, Koo TW, Shafer KE, Motz JT, Fitzmaurice M, Kramer JR, Itzkan I, Dasari RR and Feld MS: Prospects for in vivo Raman spectroscopy. Phys Med Biol 45: R1-R59, 2000.

12. Short KW, Carpenter S, Freyer JP and Mourant JR: Raman spectroscopy detects biochemical changes due to proliferation in mammalian cell cultures. Biophys J 88: 4274-4288, 2005.

13. Behl I, Kukreja L, Deshmukh A, Singh SP, Mamgain H, Hole AR and Krishna CM: Raman mapping of oral buccal mucosa: A spectral histopathology approach. J Biomed Opt 19: 126005, 2014.

14. Hu C, Wang J, Zheng C, Xu S, Zhang H, Liang Y, Bi L, Fan Z, Han B and Xu W: Raman spectra exploring breast tissues: Comparison of principal component analysis and support vector machine-recursive feature elimination. Med Phys 40: 063501, 2013.

15. Han B, Du Y, Fu T, Fan Z, Xu S, Hu C, Bi L, Gao T, Zhang H and $\mathrm{Xu} \mathrm{W}$ : Differences and relationships between normal and atypical ductal hyperplasia, ductal carcinoma in situ, and invasive ductal carcinoma tissues in the breast based on raman spectroscopy. Appl Spectrosc 71: 300-307, 2017.

16. Haka AS, Shafer-Peltier KE, Fitzmaurice M, Crowe J, Dasari RR and Feld MS: Diagnosing breast cancer by using Raman spectroscopy. Proc Natl Acad Sci USA 102: 12371-12376, 2005.

17. Haka AS, Volynskaya Z, Gardecki JA, Nazemi J, Shenk R, Wang N, Dasari RR, Fitzmaurice M and Feld MS: Diagnosing breast cancer using Raman spectroscopy: Prospective analysis. J Biomed Opt 14: 054023, 2009.

18. Hu CX, Zheng C, Zhang HP, Li-Rong BI, Shu-Ping XU, Zhi-Min F, Bing H and Qing XW: Study on fresh breast tissues by near-infrared Raman spectroscopy. Chem J Chin Univ 34: 2721-2727, 2013.

19. Yu C, Gestl E,Eckert K, Allara D and Irudayaraj J: Characterization of human breast epithelial cells by confocal Raman microspectroscopy. Cancer Detect Prev 30: 515-522, 2006.

20. Zheng C, Shao W, Paidi SK, Han B, Fu T, Wu D, Bi L, Xu W, Fan Z and Barman I: Pursuing shell-isolated nanoparticle-enhanced Raman spectroscopy (SHINERS) for concomitant detection of breast lesions and microcalcifications. Nanoscale 7: 16960-16968, 2015.

21. Zheng C, Zhang HP, Han B, Lijia L, Yabin Z, Shuping X, Heping L, Weiqing $X$ and Zhimin F: Study on fresh breast tissues by Raman spectroscopy based on robust statistics. Chem J Chin Univ 36: 74-80, 2014.

22. Naumann D: Ft-Infrared and Ft-Raman spectroscopy in biomedical research. App Spectroscopy Rev 36: 239-298, 2001.

23. Chowdary MV, Kumar KK, Kurien J, Mathew S and Krishna CM: Discrimination of normal, benign, and malignant breast tissues by Raman spectroscopy. Biopolymers 83: 556-569, 2006. 
24. Yi YN, Wang S, Gong YZ, Zheng JM, Qin J, Liang ZW and He QL: A study on biochemical constitution of human skin tissue by confocal raman microspectroscopy imaging. Acta Laser Biol Sinica 25: 391-397, 2016. (In Chinese).

25. Yu G, Lue AJ, Wang B and Zhang CZ: Raman imaging based on morphological model for human breast cancer tissues. Guang Pu Xue Yu Guang Pu Fen Xi 30: 2167-2170, 2010 (In Chinese).

26. Wang CY, Yan YG, Zhang K and Li JG: A K-nearest neighbor algorithm based on cluster in text classification. 2010 International Conference on Computer, Mechatronics, Control and Electronic Engineering (CMCE 2010) 1: 225-228, 2010.

27. Bray F, Ferlay J, Soerjomataram I, Siegel RL, Torre LA and Jemal A: Global cancer statistics 2018: GLOBOCAN estimates of incidence and mortality worldwide for 36 cancers in 185 countries. CA Cancer J Clin 68: 394-424, 2018.

28. McClatchy DM III, Zuurbier RA, Wells WA, Paulsen KD and Pogue BW: Micro-computed tomography enables rapid surgical margin assessment during breast conserving surgery (BCS): Correlation of whole BCS micro-CT readings to final histopathology. Breast Cancer Res Treat 172: 587-595, 2018.

29. Mccahill LE, Single RM, Bowles EJ, Feigelson HS, James TA, Barney T, Engel JM and Onitilo AA: Variability in Reexcision following breast conservation surgery. JAMA 307: 467-475, 2012.

30. St John ER, Al-Khudairi R, Ashrafian H, Athanasiou T, Takats Z, Hadjiminas DJ, Darzi A and Leff DR: Diagnostic Accuracy of intraoperative techniques for margin assessment in breast cancer surgery: A Meta-analysis. Ann Surg 265: 300-310, 2017.
31. Keating J, Tchou J, Okusanya O, Fisher C, Batiste R, Jiang J, Kennedy G, Nie S and Singhal S: Identification of breast cancer margins using intraoperative near-infrared imaging. J Surg Oncol 113: 508-514, 2016.

32. Blohmer JU, Tanko J, Kueper J, Gross J, Völker R and Machleidt A: MarginProbe $^{\odot}$ reduces the rate of re-excision following breast conserving surgery for breast cancer. Arch Gynecol Obstet 294: 361-367, 2016.

33. Zhang HP, Han B, Jia ZZ, Ding R, Xu B, Xu W and Fan Z: Fabrication of drug loaded fluorescent nanoparticles and its biological application in MCF-7 breast cancer cell. Chem J Chinese Univ 38: 860-865, 2017.

34. Daniel A, Prakasarao A and Ganesan S: Near-infrared Raman spectroscopy for estimating biochemical changes associated with different pathological conditions of cervix. Spectrochim Acta Part A 190: 409-416, 2018.

(c) (3) (8) This work is licensed under a Creative Commons Attribution 4.0 International (CC BY-NC 4.0) License 